Jos Zeegers

Dirk van den Ende

Cor Blom

Egbert G. Altena

Gerrit J. Beukema

Jorrit Mellema

\section{A sensitive dynamic viscometer for measuring the complex shear modulus in a steady shear flow using the method of orthogonal superposition}

Received: 19 April 1995

Accepted: 23 September 1995
J. Zeegers - Dr. D. van den Ende (凶)

C. Blom - E. G. Altena - G. J. Beukema

J. Mellema

Rheology Group

Department of Applied Physics

University of Twente

P.O. Box 217

7500 AE Enschede, The Netherlands

\begin{abstract}
A new instrument to carry out complex viscosity measurements in equilibrium and in a steady shear flow has been developed. A small amplitude harmonic excitation is superimposed orthogonally to the steady shear rate component. It is realized by a thin-walled cylinder, which oscillates in the axial direction in a rotating annular cup. The cylinder is suspended from a spring and guided by an air bearing. The spring, which itself is attached to an axially oscillating exciter, has been designed especially for a low linear and a large torsional spring constant. The motion of the cylinder and exciter is recorded and analysed. The applicable frequency ranges from 0.001 up to $50 \mathrm{~Hz}$, the steady shear rate from 0.001 up to $100 \mathrm{~s}^{-1}$ and the oscillatory shear amplitude from 3 down to 0.003 . The temperature can be set and controlled between $5^{\circ}$ and $70^{\circ} \mathrm{C}$.
\end{abstract}

The instrument is suited for viscosities in the range from $0.3 \mathrm{mPas}$ up to 1 Pas. Considering this, the range and sensitivity with respect to existing instruments has been extended significantly. Besides a description of the instrument much attention has been paid in this paper to the theoretical background of orthogonal superposition in frames of the simple fluid theory. Also the measurement range and calibration of the instrument as well as the accuracy of the obtainable results are discussed. At last some demonstration measurements on both a polymer solution and an ordering latex dispersion are presented.

Key words Superimposed oscillations - complex viscosity complex shear modulus - nearly viscometric flow - dynamic viscometer

\section{Introduction}

The viscoelastic properties of non-Newtonian fluids, like dispersions and polymers solutions depend strongly on the internal structure. For polymers, the molecular weight and configuration of the molecule chains are important parameters. In the case of, for instance, polystyrene latex dispersions the fluid consists of small charged polystyrene micro spheres dispersed in water with the addition of salt. At low excess salt concentrations the micro spheres tend to crystallize in an ordered matrix due to their mutual repulsion, and the fluid is mainly elastic at equilibrium, while at moderate concentrations the elastic properties lessen due to the weakening of the crystal.

In order to characterize such complex fluids without changing the micro structure, the response of these fluids to small harmonic shear deformations is studied. In the past, techniques have been developed for the study of fluids by this so-called dynamic method (see for instance the paper by Oka (1960) and more recently Whorlow (1992) and Marin (1988)). 
Under flow conditions the microscopic structure of the fluid may change. In the case of a polymer solution the molecule chains are stretched due to the applied shear deformation. In an ordered latex dispersion the crystal structure might break down into small fragments. It is expected that in both cases a change in the viscoelastic properties will occur.

In order to study the linear viscoelastic properties of a complex fluid while it is in a steady shear state a new torsion pendulum apparatus was developed by Van den Ende et al. (1992) a few years ago. With this apparatus a small amplitude shear deformation is superimposed parallel on a steady shear flow. This instrument operates at discrete frequencies, 86, 283, 739 and $2440 \mathrm{~Hz}$.

Experience with ordered latices, gelling materials and polymer solutions shows that it is of interest to extend the frequency range to lower frequencies $(0.01-10 \mathrm{~Hz})$. Apart from the frequency, the direction of the oscillatory shear deformation with respect to the steady shear flow can be an important aspect. Experimental considerations show that the development of a parallel superposition instrument for the low frequency range is much more complicated than an orthogonal superposition instrument. Besides, theoretical considerations show that the interpretation of the measured orthogonal complex shear modulus is less complicated as it is directly related to one material function in contrast to the parallel shear modulus which is related to two non-distinctive material functions (see for instance Huilgol (1975)).

Thus it was obviously necessary to develop an orthogonal superposition instrument. The principle of this apparatus dates back to the papers of Philippoff (1934), Birnboim and Ferry (1961) and Smith and Ferry (1949). They built instruments in which a cylindrical rod oscillates harmonically in a concentric annular beaker, containing the fluid under investigation. This arrangement with a massive oscillating geometry is called the annular pumping or Birnboim geometry. Via a mechanical or electrical impedance measurement the complex viscosity could be determined, however, it was not possible to simultaneously apply a steady shear flow to the sample. Simmons (1966) developed an instrument in which small amplitude shear deformations could be superimposed orthogonally on a steady shear flow. This was accomplished with a thin-walled cylinder oscillating axially in a concentric annular rotatable sample holder. Due to a special construction of this instrument with an "open end bottom," there is no annular pumping. A drawback is the rather large amount of sample needed (from their data we estimate this to be about $150 \mathrm{ml}$ ). Tanner and Williams (1971) improved the instrument by changing the geometry to an annular pumping set-up, thus increasing the mechanical impedance and improving the sensitivity of the instrument. Mewis and Schoukens (1978) built a similar instrument also with an annular pumping geometry.
In our group the properties of, amongst others, not too concentrated aggregating latex dispersions are studied. This system was used as a reference to set design criteria for the instrument. They concern the ranges of the deformation amplitude, frequency, viscosity and shear rate. The harmonic oscillations should not break up the structure and so the amplitude of the harmonic shear deformation $(\gamma)$ must be very small and a lower bound of approximately 0.002 is required. The frequency must range from 0.01 up to at least $10 \mathrm{~Hz}$, and over this full range, viscosities from $1 \mathrm{mPas}$ up to 1 Pas must be measured with an accuracy of $5 \%$ or better. The maximum of the shear rate $(\dot{\gamma})$ we require is of the order of $10 \mathrm{~s}^{-1}$.

In order to meet our criteria it is not possible merely to copy an existing instrument, but a new one has to be developed. The outline of this new instrument is shown in Fig. 1.

The apparatus consists of a thin-walled cylinder which oscillates harmonically in the axial direction in an annular cup which is filled with the fluid under investigation. This arrangement resembles the geometry of the instrument developed by Simmons (1966), however, now with a closed bottom. The harmonic deformation of the fluid in the cup consists of a homogeneous shear component and a small annular pumping component due to the displacement of fluid by the thin-walled cylinder. The cylinder is

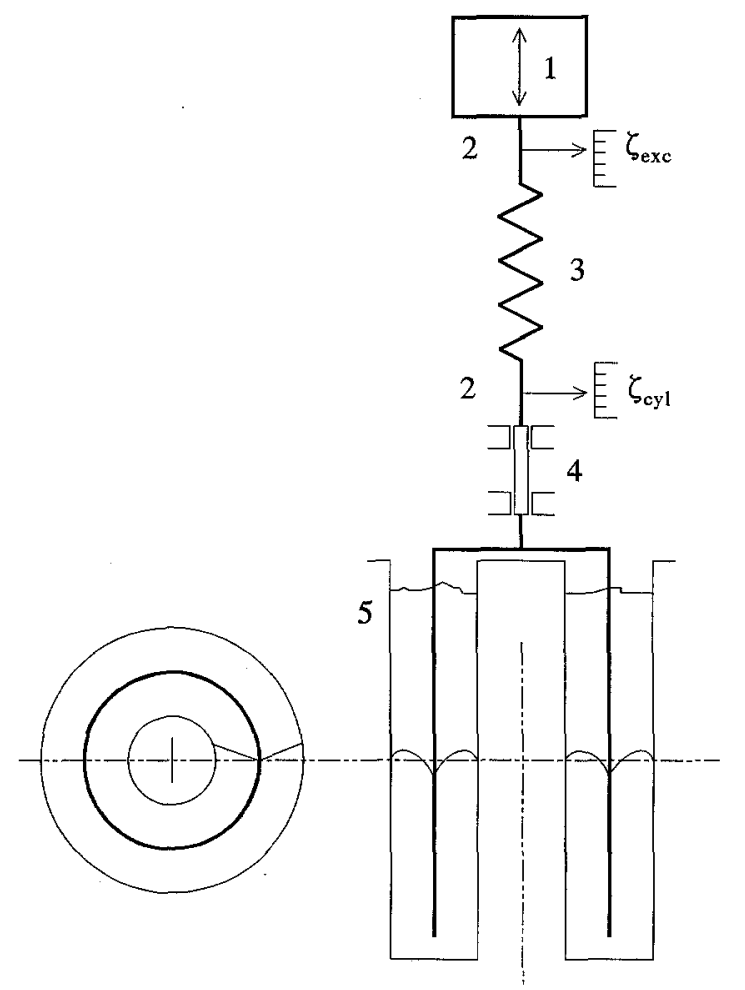

Fig. 1 Schematic outline of the instrument. 1) Exciter, 2) LVDT detectors, 3) spring, 4) air bearing, 5) measuring cylinder and annular sample holder 
suspended from a spring, which in turn is attached to an exciter. The spring has been specially designed to obtain a relatively low spring constant in the axial direction and a large torsional stiffness. The cylinder is guided axially in the vertical direction by an air bearing. A steady shear flow orthogonal to the oscillatory deformation is simultaneously applied by rotating the annular cup. The method of measurement is based on the determination of the amplitude ratio of the harmonic exciter and cylinder signals and the phase difference between these signals. From these data the fluid impedance $Z^{*}$ is calculated from which the dynamic shear modulus $G_{\perp}^{*}(\dot{\gamma}, \omega)$ or the complex viscosity $\eta_{\perp}^{*}(\dot{\gamma}, \omega)$ can be deduced.

In the paper of Tanner and Williams (1971) the theory for the orthogonal superposition is derived, however, with the assumption of a K-BKZ constitutive equation. As far as known there is no derivation of the relation between the fluid impedance $Z^{*}$ and $G_{\perp}^{*}(\dot{\gamma}, \omega)$ from the more general simple fluid theory, therefore in the next section the theory describing the measuring method of the instrument is derived. This section is divided in two subsections. In the first subsection a treatment is given to express the fluid impedance $Z^{*}$, in terms of the complex shear modulus $G_{\perp}^{*}(\dot{\gamma}, \omega)$. In the second subsection the dynamics of the instrument is considered and expressions are derived for the above-mentioned phase differences $(\varphi)$ and amplitude ratio $(R)$ in terms of the impedance $Z^{*}$. Combining these two relations eventually leads to a relation between the measured values of $(R, \varphi)$ and the shear modulus $G_{\perp}^{*}(\dot{\gamma}, \omega)$. In the third section the instrument itself, i.e., mechanical construction and electronics, is described in more detail. In the fourth section, the calibration and accuracy of the instrument are described. In the fifth section results of measurements on Newtonian and non-Newtonian fluids are presented. The paper ends with a short discussion of the results and some concluding remarks in the last section.

\section{Theory}

Relation between the shear modulus and the fluid impedance

In order to obtain an expression for the fluid impedance $Z^{*}$ in terms of the complex shear modulus $G_{\perp}^{*}(\dot{\gamma}, \omega)$ the flow field in the gap has to be calculated. This calculation consists of four parts. Part I concerns the application of simple fluid theory using material functions in order to get an expression for the memory function $\dot{G}_{\perp}(\dot{\gamma}, t)$. In part II expressions for the stresses, which make use of this memory function, are inserted in the equation of motion which leads to a differential equation describing the flow in the gap. In part III the force on the cylinder is calculated and this leads to a formal expression for the fluid impedance in terms of the complex shear modulus $G_{\perp}^{*}(\dot{\gamma}, \omega)$. In part IV a simplification is introduced by approximating the radial geometry by a two-dimensional geometry. Finally, a series expression is derived for the fluid impedance $Z^{*}$ in terms of $G^{*}{ }_{\perp}(\dot{\gamma}, \omega)$. In order to simplify the nomenclature the " $\perp$ " index will be omitted in the further analysis. Unless otherwise indicated it will be assumed that with $G^{*}, \eta^{*}$ or $\dot{G}$ the orthogonal quantities $G_{\perp}^{*}(\dot{\gamma}, \omega), \eta_{\perp}^{*}(\dot{\gamma}, \omega)$ or $\dot{G}_{\perp}(\dot{\gamma}, t)$ are meant.

I) The shear flow in the gap due to the rotating cup is considered as a viscometric flow, on which a small amplitude oscillatory flow is superimposed orthogonally. This results in a nearly viscometric flow as defined by Pipkin and Owen (1967). For such a flow the stress can be expressed as

$$
\underline{\underline{T}}(t)=-\left(p_{0}+\tilde{p}(t)\right) \underline{\underline{\delta}}+{ }^{0} \underline{\underline{\underline{T}}}+\underset{\underline{\underline{T}}}{\tilde{T}}(t),
$$

where $\left(-p_{0} \delta+{ }^{0} T\right)$ is the purely viscometric part and $(-\tilde{p}(t) \delta+\tilde{\underline{T}}(t))$ the nonviscometric oscillatory part of the stress (see Schowalter, 1978). The nonviscometric deviatoric stress $\tilde{\underline{T}}$ can be written as

$$
\tilde{\underline{T}}(t)=\int_{-\infty}^{\infty} \underline{\underline{K}}\left({ }^{0} \underline{\underline{E}}_{t} ; s\right): \underline{\underline{E_{t}}}(t-s) d s
$$

Here $E_{t}(t-s)$ is the strain tensor at time $t-s$ relative to time $\overline{\bar{t}},{ }^{0} E_{t}(t-s)$ its viscometric part and $\tilde{E}_{t}(t-s) \equiv$ $\underline{E}_{i}(t-s)-{ }^{0} E_{t}(t-s)$ its oscillatory part. $K$ is a fourth order tensor and the: indicates a double contraction. Here the notation used by Van den Ende et al. (1992) is followed. They discussed the same problem for parallel superposition. For our cylindrical geometry the flow field expressed on an orthogonal cylindrical basis $\left\{\underline{e}_{\varphi}, \underline{e}_{r}, \underline{e}_{z}\right\}$ is given by

$$
\begin{aligned}
& \varphi\left(t^{\prime}\right)=\varphi(t)+\left(t^{\prime}-t\right) f(r) \\
& r\left(t^{\prime}\right)=r(t) \\
& z\left(t^{\prime}\right)=z(t)+\zeta\left(t^{\prime}, r\right)-\zeta(t, r) .
\end{aligned}
$$

Here $f(r)$ is the angular velocity at a distance $r$ from the axis. Furthermore $\zeta(t, r)$ represents the axial displacement at $r$ on time $t$ due to the harmonic oscillation. Evaluation of the relative strain results in

$$
{ }^{0} \underline{E}_{t}(t-s)=-\frac{1}{2} s \dot{\gamma}\left(\underline{e}_{r} \underline{e}_{\varphi}+\underline{e}_{\varphi} \underline{e}_{r}\right)+\frac{1}{2} s^{2} \dot{\gamma}^{2} \underline{e}_{r} \underline{e}_{r}
$$

and

$$
\widetilde{E}_{\underline{t}}(t-s)=\frac{1}{2} \frac{\partial}{\partial r}(\zeta(t-s, r)-\zeta(t, r))\left(\underline{e}_{z} \underline{e}_{r}+\underline{e}_{r} \underline{e}_{z}\right),
$$

where $j=r \partial f / \partial r$ is the steady shear rate, which is slightly 
$r$-dependent. The base vectors $\underline{e}_{r}$ and $\underline{e}_{\varphi}$ are considered at time $t$. For the calculations the $T_{z r}$ and $T_{z z}$ component of the stress are important as they determine the axial component of the fluid motion. First the viscometric part is considered and it follows that ${ }^{0} T_{z r}=0$ because $\left({ }^{0} E_{t}\right)_{z r}=0$, but ${ }^{0} T_{z z}$ is not necessarily equal to zero due to eventual normal stress differences (see Schowalter, 1978). So in the viscometric case $T_{z z}=-p_{0}+{ }^{0} T_{z z}$ and $T_{z r}=0$. The oscillatory parts are calculated from Eqs. (2), (4) and (5). Using the symmetry relation $K_{z r z r}=K_{z r r z}$ (see Huilgol, 1975) a memory function $\dot{G}$ can be defined by:

$$
\dot{G}(\dot{\gamma}, s) \equiv K_{z r z r}(\dot{\gamma}, s)-\delta(s) \cdot \int_{\infty}^{\infty} K_{z r z r}\left(\dot{\gamma}, s^{\prime}\right) d s^{\prime} .
$$

II) Now expressions will be given for the stresses in the $z$-direction. From Eq. (6) an expression can be given for the shear stress in the $z$-direction due to the radial deformation profile:

$$
T_{z r}(t)=\int_{-\infty}^{\infty} \dot{G}(\dot{\gamma}, s) \frac{\partial}{\partial r} \zeta(t-s, r) d s .
$$

From the relation $K_{z z z r}=K_{z z r z}=0$ (see Huilgol, 1975), one obtains $\tilde{T}_{z z}(t)=0$ so:

$$
T_{z z}(t)=-p_{0}+{ }^{0} T_{z z}+\tilde{p}(t)
$$

Considering a ring-shaped fluid element with volume $2 \pi r d r d z$, the equation of motion in the axial direction for this ring can be obtained from Eqs. (7) and (8),

$$
\begin{aligned}
\rho \frac{\partial^{2}}{\partial t^{2}} \zeta(t, r)= & \frac{1}{r} \frac{\partial}{\partial r}\left[r \int_{\infty}^{\infty} \dot{G}(\dot{\gamma}, s) \frac{\partial}{\partial r} \zeta(t-s, r) d s\right] \\
& +\left(\frac{\partial}{\partial z} T_{z z}-\rho g\right) .
\end{aligned}
$$

In the viscometric case, in which $\zeta=0$, one observes from Eq. (9) that $\left(\frac{\partial}{\partial z} T_{z z}-\rho g\right)=0$ and so $\frac{\partial}{\partial z}\left({ }^{0} T_{z z}-\rho_{0}\right)=\rho g$. Taking the Fourier transform of Eq. (9) the following result is obtained:

$$
-\omega^{2} \rho \hat{\zeta}(\omega, r)=\frac{1}{r} \frac{\partial}{\partial r}\left(r G^{*}(\dot{\gamma}, \omega) \frac{\partial}{\partial r} \xi(\omega, r)\right)-\frac{\partial \hat{p}}{\partial z},
$$

where $\hat{\zeta}$ denotes the transform of $\zeta(t)$ and $\hat{p}$ that of $\tilde{p}(t)$. The complex shear modulus, $G^{*}(\dot{\gamma}, \omega)$, is now defined as the Fourier transform of the memory function $\dot{G}(\dot{\gamma}, t)$ as defined in Eq. (6). In the apparatus the gap width is very small with respect to the radius of the rotating cup. In that case and if the fluid is not extremely shear thinning the $r$-dependence of $\dot{\gamma}$ can be neglected and Eq. (10) reduces to:

$$
\frac{1}{r} \frac{\partial}{\partial r}\left(r \frac{\partial \hat{\zeta}}{\partial r}\right)+k^{* 2} \hat{\zeta}=k^{* 2} \hat{\zeta}_{p}
$$

with $k^{*} \equiv\left(\omega^{2} \rho / G^{*}\right)^{1 / 2}$ and $\hat{\zeta}_{p} \equiv(\partial \hat{p} / \partial z) /\left(\omega^{2} \rho\right)$, which is a particular solution of Eq. (11) because the oscillatory part of the isotropic pressure $\hat{p}$ is independent of $r$. Equation (11) is the differential equation which describes the harmonic flow in the gap.

III) In Fig. 2 one side of the geometry of the fluid-filled gap is shown in cross-section. Here the gap width is displayed disproportionately larger than the radius of the measurement cup and cylinder.

In the Figure $a$ and $d$, and $b$ and $c$ are the inner and outer walls of the annular cup and cylinder, respectively and $\zeta_{c}$ is the axial displacement of the cylinder. In the inner and outer gap the global characteristics of the deformation flow profile are sketched. Due to the harmonic motion of the cylinder there is an approximately linear shear deformation profile (upper drawing). In addition there is a pressure-driven counter flow, which is represented by the parabolic deformation profile. The amount of

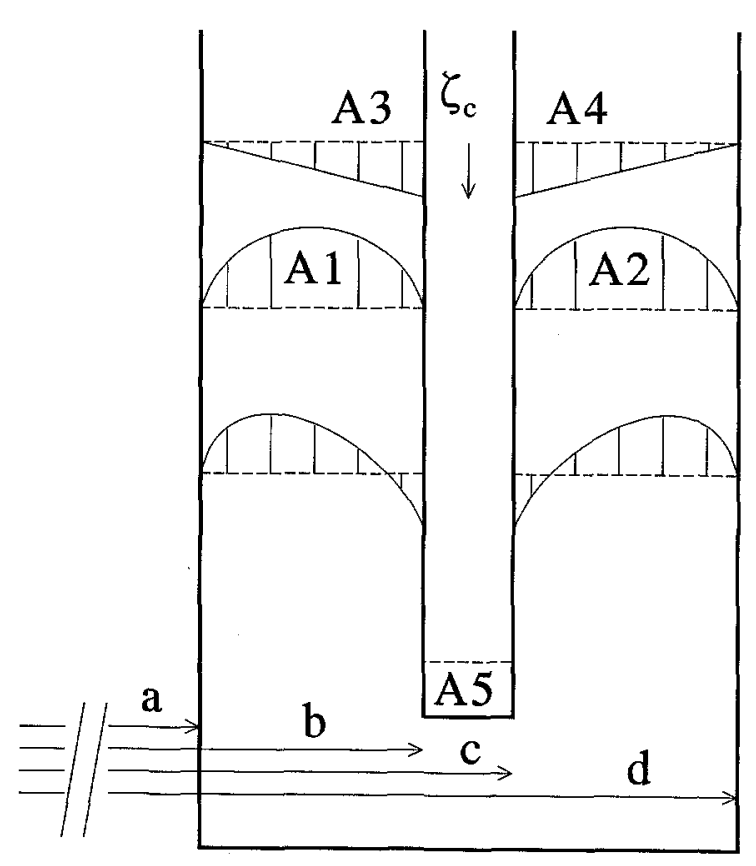

Fig. 2 Cross-section of the measurement geometry with the flow profile. To conserve mass, the area $A 1+A 2$ must be equal to $A 3+A 4+A 5$. Also the radii $a, b, c$ and $d$ are indicated 
this counter flow depends on the thickness of the cylinder and the width of the gap and it is caused by the displacement of fluid at the bottom. The addition of both profiles yields the total flow profile shown in the figure.

The differential Eq. (11) has to be solved in agreement with the boundary conditions, given by the no-slip condition on the walls,

$$
\begin{aligned}
& \hat{\zeta}(a)=\hat{\zeta}(d)=0 \\
& \hat{\zeta}(b)=\hat{\zeta}(c)=\zeta_{c} .
\end{aligned}
$$

Another boundary condition results from the conservation of fluid mass, i.e., the volume of displaced fluid in both gaps is equal to the volume displaced by the cylinder. From this condition the ratio $Q \equiv \hat{\zeta}_{p} / \zeta_{c}$ can be determined.

Solving the homogeneous part of Eq. (11), one can obtain for $a \leq r \leq b$ the formal solutions $\xi_{1}(r)$ and $\xi_{2}(r)$ which fulfill the boundary conditions: $\xi_{1}(a)=1$, $\xi_{1}(b)=0, \xi_{2}(a)=0$ and $\xi_{2}(b)=1$. With these solutions and the particular solution the solution $\hat{\zeta}_{1}(r)$ of Eq. (11) which fulfills the boundary conditions (16) in the inner gap $a \leq r \leq b$ is given by

$$
\zeta_{1}(r)=\zeta_{c}\left(Q-Q \xi_{1}(r)+(1-Q) \xi_{2}(r)\right)
$$

For $c \leq r \leq d$ the formal dimensionless solutions $\xi_{3}(r)$ and $\xi_{4}(r)$ for the homogeneous part of Eq. (11) are obtained which fulfill the boundary conditions: $\xi_{3}(c)=1$, $\xi_{3}(d)=0, \xi_{4}(c)=0$ and $\xi_{4}(d)=1$. The solution $\bar{\xi}_{2}(r)$ of Eq. (11) which fulfills the boundary conditions (12) in the outer gap $c \leq r \leq d$ is given by

$$
\hat{\zeta}_{2}(r)=\zeta_{c}\left(Q-Q \xi_{4}(r)+(1-Q) \xi_{3}(r)\right) .
$$

The final solutions $\hat{\zeta}_{1}$ and $\hat{\xi}_{2}$ are equal to the total flow profile in Fig. 2.

In Eqs. (13) and (14) the value of $Q$ is still an unknown constant which has to be determined from the conservation of mass:

$$
2 \pi \int_{a}^{b} \widehat{\zeta}_{1}(r) r d r+2 \pi \zeta_{c} \int_{b}^{c} r d r+2 \pi \int_{c}^{d} \hat{\zeta}_{2}(r) r d r=0 .
$$

In order to evaluate Eq. (15) the following integrals are defined:

$$
\begin{aligned}
& I_{1} \equiv \int_{a}^{b} \xi_{1}(r) r d r, I_{2} \equiv \int_{a}^{b} \xi_{2}(r) r d r, I_{3} \equiv \int_{c}^{d} \xi_{3}(r) r d r \text { and } \\
& I_{4} \equiv \int_{c}^{d} \xi_{4}(r) r d r
\end{aligned}
$$

Substitution of Eq. (13) and (14) in Eq. (15) gives with the application of Eq. (16)

$$
Q=\frac{I_{2}+I_{3}+\left(c^{2}-b^{2}\right) / 2}{I_{1}+I_{2}+I_{3}+I_{4}-\left(d^{2}-c^{2}+b^{2}-a^{2}\right) / 2} .
$$

Now it is possible to calculate the total axial oscillatory force acting on the cylinder. This force $\hat{F}_{f l}$ consists of two parts, a shear force and a buoyancy force. The shear force $\hat{F}_{\text {sh }}$ can be calculated by

$$
\hat{F}_{s h}=2 \pi L G *\left(\left.c \frac{\partial}{\partial r} \hat{\zeta}_{2}\right|_{r=c}-\left.b \frac{\partial}{\partial r} \hat{\zeta}_{1}\right|_{r=b}\right) .
$$

The oscillatory buoyancy force $\hat{F}_{b}=-\pi\left(c^{2}-b^{2}\right) L(\partial \hat{p} /$ $\partial z$ ) is given by

$$
\hat{F}_{b}=-\pi\left(c^{2}-b^{2}\right) L \omega^{2} \rho Q \zeta_{c} .
$$

Here $L$ is the length of the fluid column along the cylinder. Besides this there is also a stationary buoyancy force due to the viscometric part of the flow,

$$
F_{b}^{\text {(stat) }}=\pi\left(c^{2}-b^{2}\right) L \rho g .
$$

This force determines the equilibrium position of the cylinder and has no influence upon the calculation of the impedance.

The complex impedance $Z^{*}$ is defined as $Z^{*}=-\hat{F}_{f l} / \hat{v}_{c}$, where $\hat{v}_{c}=i \omega \zeta_{c}$ is the axial velocity of the cylinder. $Z^{*}$ is calculated using Eqs. (18) and (19)

$$
\begin{aligned}
Z^{*}= & \frac{2 \pi L G}{i \omega}\left(\frac{1}{2}\left(c^{2}-b^{2}\right) k^{* 2} Q+b \frac{\partial}{\partial r} \hat{\zeta}_{1} /\left.\zeta_{c}\right|_{r=b}\right. \\
& \left.-c \frac{\partial}{\partial r} \hat{\zeta}_{2} /\left.\zeta_{c}\right|_{r=c}\right) .
\end{aligned}
$$

With Eq. (21) the relation between $Z^{*}$ and $G *$ has been obtained formally. If the solutions $\xi_{1}(r), \xi_{2}(r), \xi_{3}(r)$ and $\xi_{4}(r)$ are known, $Z^{*}$ can actually be calculated.

IV) Formally the solutions $\xi_{i}$, with $i \in\{1,2,3,4\}$, can be expressed in Bessel functions of the first and second type of order zero, $J_{0}$ and $Y_{0}$ respectively:

$$
\xi_{i}(r)=\alpha_{\mathrm{i}} J_{0}\left(k^{*} r\right)+\beta_{i} Y_{0}\left(k^{*} r\right) .
$$

A full analysis of the problem in terms of $J_{0}\left(k^{*} r\right)$ and $Y_{0}\left(k^{*} r\right)$ results in a rather tedious calculation. This analysis has been given by Markovitz (1952). However, the functions $\xi_{i}$ can be calculated rather easily numerically for given $G^{*}$. Moreover for our geometry, where $(b-a)=(c-d) \ll a$, the curvature of the cylindrical surface can be ignored and the homogeneous part of Eq. (11) can be approximated by 


$$
\frac{\partial^{2}}{\partial r^{2}} \hat{\zeta}+k^{* 2} \bar{\zeta}=0
$$

In that case the functions $\xi_{i}$ are given by

$$
\begin{aligned}
& \xi_{1}(r)=\frac{\sin \left(k^{*}(b-r)\right)}{\sin \left(k^{*}(b-a)\right)}, \quad \xi_{2}(r)=\frac{\sin \left(k^{*}(r-a)\right)}{\sin \left(k^{*}(b-a)\right)}, \\
& \xi_{3}(r)=\frac{\sin \left(k^{*}(d-r)\right)}{\sin \left(k^{*}(d-c)\right)}, \quad \xi_{4}(r)=\frac{\sin \left(k^{*}(r-c)\right)}{\sin \left(k^{*}(d-c)\right)}
\end{aligned}
$$

while conservation of mass now leads to

$$
\int_{a}^{b} \hat{\zeta}_{1}(r) d r+\zeta_{c} \int_{b}^{c} d r+\int_{c}^{d} \hat{\zeta}_{2}(r) d r=0
$$

This results for the ratio $Q=\widehat{\zeta}_{p} / \zeta_{c}$ in

$$
Q=\frac{1-\cos (q)+\varepsilon q \sin (q)}{2(1-\cos (q))-q \sin (q)},
$$

with $q \equiv k^{*}(b-a)$ and $\varepsilon \equiv(c-b) / 2(b-a)$. The impedance is calculated from Eq. (21):

$$
\begin{aligned}
Z^{*}= & \frac{2 \pi L(b+c) G^{*}}{i \omega(b-a)} \\
& \times\left(q^{2} Q\left(\frac{1-\cos (q)}{q \sin (q)}+\varepsilon\right)+\frac{q^{2} \cos (q)}{q \sin (q)}\right)
\end{aligned}
$$

From this equation it is possible to calculate $Z^{*}$ if one knows $G^{*}$, however, in the experiment $Z^{*}$ is determined from a measured phase difference $(\varphi)$ and amplitude ratio $(R)$, and $G^{*}$ has to be calculated. This is difficult with Eq. (27), because there is no unambiguous inverse relation $G^{*}\left(Z^{*}\right)$. But since the apparatus operates in the so-called gap-loading limit, i.e., $q \ll 1$, Eqs. (26) and (27) are developed in powers of $q^{2}$ which finally leads to:

$$
\begin{aligned}
Z^{*}= & \frac{U G^{*}}{i \omega}\left(1-\frac{\alpha_{1}}{\alpha_{0}} \frac{\rho \omega^{2}(b-a)^{2}}{G^{*}}-\frac{\alpha_{2}}{\alpha_{0}} \frac{\rho^{2} \omega^{4}(b-a)^{4}}{G^{* 2}}\right. \\
& \left.-\frac{\alpha_{3}}{\alpha_{0}} \frac{\rho^{3} \omega^{6}(b-a)^{6}}{G^{* 3}}-. .\right)
\end{aligned}
$$

with

$$
U=2 \pi L \frac{(b+c)}{(b-a)} \alpha_{0}
$$

and

$$
\begin{array}{ll}
\alpha_{0}=\left(4+12 \varepsilon+12 \varepsilon^{2}\right) & \alpha_{2}=\left(11+9 \varepsilon+9 \varepsilon^{2}\right) / 6300 \\
\alpha_{1}=\left(2+3 \varepsilon+18 \varepsilon^{2}\right) / 15 & \alpha_{3}=\left(7+3 \varepsilon+3 \varepsilon^{2}\right) / 189000
\end{array}
$$

where $\varepsilon=(c-b) /(2(b-a))$.

The first order approximation, using only the terms with coefficients $\alpha_{0}$ and $\alpha_{1}$, represents the gap loading approximation and gives a simple relation between $G^{*}$ and $Z^{*}$ :

$$
G^{*}=\frac{i \omega Z^{*}}{U}+\frac{\alpha_{1}}{\alpha_{0}} \rho(b-a)^{2} \omega^{2}
$$

Also an expression for the second order correction term, using the $\alpha_{2}$ term, in case of nearly surface loading is derived:

$$
\Delta G^{*}=\frac{\alpha_{2}}{\alpha_{0}}\left(\rho(b-a)^{2} \omega^{2}\right)^{2} \frac{1}{G^{*}}
$$

Equation (31) is used in the further analysis, while Eq. (32) is used to check the gap-loading condition in the experiments, which is discussed in the fourth section.

In the analysis the influence of the finite length of the cylinder, so-called end effect, is not considered in detail, but as long as the instrument operates in the gap-loading limit it can be shown by considering the Stokes equations for creeping flow that the shear stresses as well as the pressure gradients are proportional to $\eta^{*}$ or $G^{*}$. Thus the end contributions to $Z^{*}$ are also proportional to $G^{*}$, so they are absorbed in the geometrical constant $U$. From the calibration procedure, discussed in the fourth section, it will become clear that this effect is a few percent at most.

The impedance and the measured amplitude ratio and phase difference

To obtain the relation between the impedance and the measured amplitude ratio and phase difference, in this section the equation of motion of the measuring cylinder is considered. The forces acting in axial direction on the cylinder are: the force exerted by the fluid $F_{f l}$, the force exerted by the spring $F_{s p}$, the force due to damping in the bearing $F_{d}$, the gravitational force $F_{g}$ and the buoyancy forces $F_{b}$. In case no oscillation is superimposed, there are static forces only, which sum must be zero,

$$
F_{b}^{\text {(stat) }}+F_{s p}^{\text {(stat) }}+F_{g}=0 .
$$

When a small amplitude oscillation is present, the fluid force is given by

$$
F_{f l}(t)=-\int_{-\infty}^{\infty} Z(\dot{\gamma}, s) \frac{\partial}{\partial t} \zeta_{c}(t-s) d s+F_{b}^{\text {(stat })}
$$


where $Z(\dot{\gamma}, t)$ is the inverse Fourier transform of $Z^{*}(\dot{\gamma}, \omega)$ as derived in the second section and $F_{b}^{\text {(stat) }}$ is the static buoyancy force as given in Eq. (20). Taking into account the mass of the spring $m$, the spring force consists of a static and an oscillatory part: $F_{s p}=F_{s p}^{\text {(stat) }}+\widetilde{F}_{s p}(t)$ where the oscillatory part is given by its Fourier transform $\hat{F}_{s p}(\omega)=\mathscr{F}\left\{\tilde{F}_{s p}(t)\right\}$

$$
\hat{F}_{s p}(\omega)=K \frac{\left(\omega / \omega_{s}\right)}{\sin \left(\omega / \omega_{s}\right)}\left(\hat{\zeta}_{m}-\hat{\zeta}_{c} \cos \left(\omega / \omega_{s}\right)\right)
$$

with $K$ the spring constant and $\omega_{s} \equiv(K / m)^{1 / 2}$. $\xi_{m}$ is the displacement of the exciter side of the spring and $\zeta_{c}$ the oscillatory displacement of the cylinder. In this equation the inertia of the spring is taken into account. The force due to the damping in the bearing is given by

$$
F_{d}=-D \frac{\partial}{\partial t} \zeta_{c}
$$

In this equation $D$ is the damping coefficient. The gravity force is simply

$$
F_{g}=-M g \text {, }
$$

where $M$ is the mass of the cylinder and the moving part of the air bearing and $g$ the gravity constant. From Eq. (33) the equilibrium position of the spring is obtained, which can depend on $\dot{\gamma}$. The displacement $\zeta_{c}$ is measured with respect to this equilibrium position. Now in the frequency domain the equation of motion reads

$$
\begin{aligned}
-\omega^{2} M \hat{\zeta}_{c}= & K \frac{\left(\omega / \omega_{s}\right)}{\sin \left(\omega / \omega_{s}\right)}\left(\hat{\zeta}_{m}-\hat{\zeta}_{c} \cos \left(\omega / \omega_{s}\right)\right) \\
& -i \omega\left(D+Z^{*}\right) \hat{\zeta}_{c}
\end{aligned}
$$

From Eq. (38) $Z^{*}$ can be expressed in $\hat{\zeta}_{m} / \hat{\zeta}_{c}=\operatorname{Re}^{i \varphi}$, where $R$ is the amplitude ratio between the exciter and cylinder signal and $\varphi$ is the phase difference between these signals. Applying these definitions leads to

$$
\begin{aligned}
i \omega Z^{*}= & K \frac{\left(\omega / \omega_{s}\right)}{\sin \left(\omega / \omega_{s}\right)}\left(\operatorname{Re}^{i \varphi}-\cos \left(\omega / \omega_{s}\right)\right) \\
& -i \omega D+\omega^{2} M
\end{aligned}
$$

As discussed in the second section, Eq. (31) is applied and by inserting this in Eq. (39) it can be shown that $G^{*}$ is related to $\operatorname{Re}^{i \varphi}$ according to

$$
\begin{aligned}
G^{*}= & \frac{1}{U}\left(K \frac{\omega / \omega_{s}}{\sin \left(\omega / \omega_{s}\right)}\left(\operatorname{Re}^{i \varphi}-\cos \left(\omega / \omega_{s}\right)\right)-i \omega D\right) \\
& +\frac{M^{\prime}}{U} \omega^{2}
\end{aligned}
$$

with $M^{\prime} \equiv M+\rho U \alpha_{1}(b-a)^{2} / \alpha_{0}$. Here it has to be remarked that terms up to and including $\alpha_{1}$ are taken into account and higher orders are ignored.

After calibration of the parameters $\omega_{s}, K / U, D / U$ and $M^{\prime} / U$, Eq. (40) will be used to calculate $G^{*}$ and $\eta^{*}=G^{*} /(i \omega)$ from the measured phase difference $\varphi$ and amplitude ratio $R$.

\section{Description of the instrument}

Mechanical design

Figure 1 gives a schematic diagram of the apparatus. In this diagram six important parts can be distinguished. These are:

1) Exciter-unit, which is in fact the motor for the harmonic motion.

2) Displacement transducers to measure the cylinder and exciter motion.

3) The spring attached to the exciter, which supports the cylinder.

4) Air bearing which guides the vertical motion of the cylinder.

5) Cylinder, annular cup and thermostat jacket assembly.

6) Motor which is used to rotate the annular cup, not shown in Fig. 1.

The exciter and displacement transducers will be discussed in the next section.

For the spring a special cylindrical zigzag lamella construction has been chosen. It is schematically shown in Fig. 3 and consists of circular lamellae which are con-

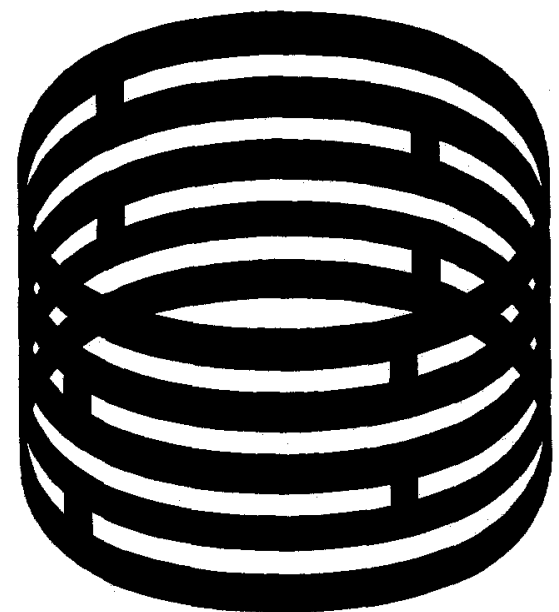

Fig. 3 Schematic representation of the spring in projection 
nected to the next lamella at two diagonal opposing positions. The interlinking positions jump each time with $\pi / 2 \mathrm{rad}$. In this way units with four $\pi / 2$ quarter circular lamella forming a link are connected to each other.

Depending on the material, lamella thickness and width, and diameter, each link has a fixed spring constant, which can be calculated easily with engineering formulae, if one knows the Young's modulus of the material. Furthermore, by connecting a number of links together any arbitrary spring constant can be arranged, but practically the value ranges from 5 up to $1000 \mathrm{~N} / \mathrm{m}$. Due to the geometry, which has been obtained by spark corrosion of a tube, the spring is rigid with respect to a torque. This has the advantage that now there are no special interventions necessary to prevent rotation of the cylinder during a steady shearing experiment. The spring constant of our system is approximately $75 \mathrm{~N} / \mathrm{m}$ and the torsional constant is $0.025 \mathrm{Nm} / \mathrm{rad}$. Shear experiments can be carried out at maximum shear rate $\left(100 \mathrm{~s}^{-1}\right)$ with fluids with a viscosity up to 0.2 Pas. To avoid torsional overload of the spring with fluids with a larger viscosity in shear experiments, a rotation protection arrangement has been applied on the air bearing. In order to increase the sensitivity at low frequencies the spring can easily be exchanged by a type with a lower spring constant. The characteristic resonance frequency of the cylinder and spring system in air is approximately $7.3 \mathrm{~Hz}$, and can be decreased in this way to a minimum of $2 \mathrm{~Hz}$.

The radial air bearing is necessary to guide the cylinder exactly in the vertical direction and fixing it radially. The bearing consists of two stators and a sliding shaft. The stators are axially aligned in a cylindrical housing and spaced with a distance of approximately $45 \mathrm{~mm}$ from each other. They are designed following the rules as given in Powell (1970). Some relevant data are listed in Table 1.

The viscous damping of the air bearing has been measured and is in good agreement with the theoretical estimation of the viscous air damping in the clearance between stator and the sliding shaft. This damping is equivalent with the damping of a $0.03 \mathrm{mPas}$ fluid in the measuring geometry.

The cylinder has been made of commercially available stainless steel precision tubing. It is possible to disconnect it from the sliding shaft of the air bearing. The connection is made with a fitting edge which is locked with a nut and screw thread system. Important to note here is that

Table 1 Characteristic data for each stator of the air bearing

\begin{tabular}{lc}
\hline Shaft diameter & $8 \mathrm{~mm}$ \\
Stator length & $8 \mathrm{~mm}$ \\
Gap width & $15 \mu \mathrm{m}$ \\
Number of holes/ring & 8 \\
Hole diameter & $0.20 \mathrm{~mm}$ \\
Excess pressure & $1 \mathrm{bar}$ \\
Maximum radial thrust & $\simeq 1.5 \mathrm{~N}$ \\
\hline
\end{tabular}

the cylinder is suspended from three thin $(1 \mathrm{~mm})$ bars. The sample fluid level lies just at the position of the bars and in this way the influence of capillary forces due to irregular wetting is diminished. Dimensions of the cylinder are shown in Table 2.

The annular measurement cup, which is part of the thermostat jacket assembly, fits closely on a hollow shaft which is driven by a pulley and motor unit in order to rotate the cup to apply a steady shear rate. The cup can be removed easily for the cleaning and changing of fluid samples. By a special through flow system using the hollow shaft, the inner as well as the outer wall of the annular cup can be thermostated very well within $0.05^{\circ} \mathrm{C}$.

The annular cup has been constructed on a precision lathe with an accuracy of $5 \mu \mathrm{m}$. Some characteristic dimensions of the cup are listed in Table 2. As mentioned above, much care has been taken to align and position the cylinder as well as possible in the annular cup. Axial alignment is by estimation within approximately $0.02 \mathrm{~mm}$ correct, but the precision tubing itself is liable to manufacturing inaccuracies in the radial direction of maximal $\pm 0.025 \mathrm{~mm}$.

The apparatus is mounted on a frame and the assembly of exciter, displacement transducers, spring and cylinder can be lifted with a pneumatic system in order to raise the cylinder out of the annular cup. This is necessary to supply and remove a liquid sample. Much attention was paid to the frame and guiding mechanisms in order to get a rigid construction. No special steps were taken for damping isolation of external (building) vibrations.

\section{Electronic design}

The electronic design is presented with reference to Fig. 4. In this diagram the main electronic detectors and components are shown schematically. From above to below the following components can be distinguished:

The exciter which drives the spring and cylinder system is a mini-shaker (Bruël \& Kjær, type 4810). The displacement of the cylinder and the exciter is recorded with Linear Variable Differential Transformer (LVDT) units

Table 2 Numerical values of the dimensions of the instrument

\begin{tabular}{llc}
\hline Inner radius cup & $a$ & $8.05 \mathrm{~mm}$ \\
Inner radius cylinder & $b$ & $8.76 \mathrm{~mm}$ \\
Outer radius cylinder & $c$ & $8.99 \mathrm{~mm}$ \\
Outer radius cup & $d$ & $9.70 \mathrm{~mm}$ \\
Gap width & $b-a$ & $0.71 \mathrm{~mm}$ \\
Cylinder wall thickness & $c-b$ & $0.23 \mathrm{~mm}$ \\
Cylinder length & $L$ & $100 \mathrm{~mm}$ \\
Cylinder mass & $M$ & $18 \mathrm{~g}$ \\
Vibrating mass & $M$ & $35 \mathrm{~g}$ \\
Spring constant & $K$ & $75 \mathrm{~N} / \mathrm{m}$ \\
Amount of sample needed & & $9 \mathrm{ml}$ \\
System resonance frequency & & $7.3 \mathrm{~Hz}$ \\
\hline
\end{tabular}




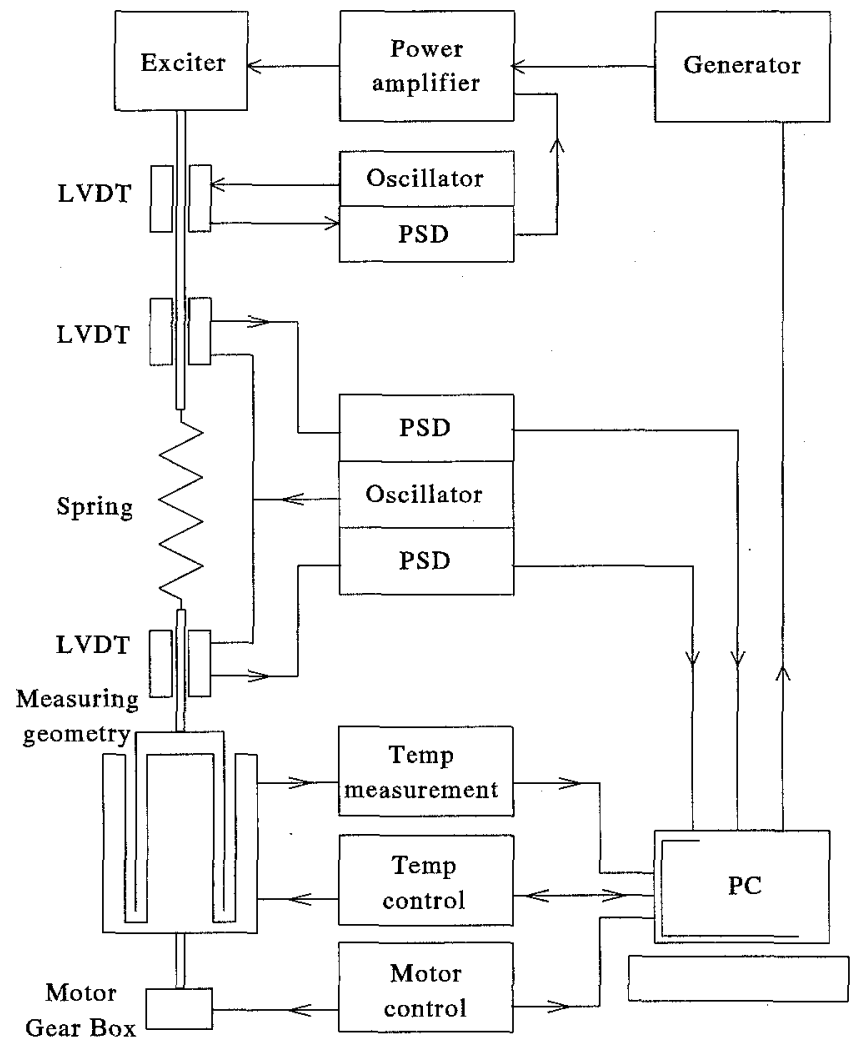

Fig. 4 Electronics in a simplified block diagram

(Lucas Schaevitz, 050 HR). These devices have been chosen to attain a high displacement resolution.

A through-flow thermostat system (Haake F-3 K) controls the temperature of the fluid in the jacket. A PRT-100 is used to measure the temperature.

A dc motor (Electro-Craft, S19-1A/T), in combination with a gear box $(100: 1$ or $10000: 1)$ and belt, is used to drive the rotational motion of the annular cup.

For the measurement and control-functions standard laboratory apparatus has been used. An exception is the control of the exciter, which is part of a closed loop control unit in order to correct for non-linearities of the exciter. As a signal conditioner a two-channel PSD-detector with a common oscillator is used (Schlumberger CAH-911-001-8D). This system has enough bandwidth $(500 \mathrm{~Hz})$, and has the advantage that due to the symmetry the influence of temperature gradients is minimized. Finally, a personal computer (Olivetti M240) is used to control the instrument and carry out the data handling. In this way measurements can be run without human intervention.

The long time stability of the LVDT performance is mainly determined by slow temperature fluctuations in the laboratory. To attain a small temperature dependency of the LVDT's, it appeared that the in situ measurement

of the self-induction $(L)$ and the electric quality factor $(Q)$ of the primary windings was a feasible selection criterion. If the mutual differences in $L$ were less than $0.5 \%$ and in $Q$ smaller than $2 \%$, the LVDT's did not dominate the temperature behavior of the instrument.

The following temperature coefficients for the amplitude ratio and phase have finally been obtained:

$$
\begin{aligned}
& \frac{1}{R} \frac{d R}{d T} \simeq 6 \cdot 10^{-5}{ }^{\circ} \mathrm{C}^{-1} \\
& \frac{d \varphi}{d T}<5 \cdot 10^{-6} \mathrm{rad} /{ }^{\circ} \mathrm{C} .
\end{aligned}
$$

Here $T$ is the temperature in the laboratory. In order to reduce the temperature differences between the LVDT's, a draught-screen has been applied around the LVDT's and the spring. This resulted in a reduction of the shorttime noise with a factor $3-5$. The characteristic mechanical noise in the amplitude due to vibrations, as measured with the LVDT transducers, is approximately $300 \mathrm{~nm}$. This was measured in the situation where the cup was filled with ethanol.

For each measurement exactly one sine period of the LVDT signals is sampled and stored. With a cross-correlation technique the amplitude ratio $(R)$ and phase difference $(\varphi)$ between cylinder and exciter motion are determined. Measurements can be performed for any number of sine wave periods. A special amplitude setting procedure is available to realize the desired cylinder amplitude. Some characteristic numerical data of the measurement system are listed in Table 3.

\section{Calibration and measurement range}

\section{Calibration}

Earlier, Eq. (40) was given, which is used to calibrate the instrument. Rewriting this equation and considering the case of a Newtonian fluid with $G^{\prime}=0$ and furthermore $G^{\prime \prime}=\eta^{\prime} \omega$, this leads to:

$$
\begin{aligned}
& R \cos \varphi=\cos \left(\omega / \omega_{s}\right)-C_{1} \omega \cdot \sin \left(\omega / \omega_{s}\right) \\
& R \sin \varphi=\left(C_{2} \eta^{\prime}+C_{3}\right) \cdot \sin \left(\omega / \omega_{s}\right)=C_{0} \cdot \sin \left(\omega / \omega_{s}\right),
\end{aligned}
$$

Table 3 Control and measure ranges for the experimental parameters

Frequency

Excitator amplitude

Rotation

Sample temperature
$0.001-50 \mathrm{~Hz}$

$0.5-500 \mu \mathrm{m}$

$0.0005-10 \mathrm{rad} / \mathrm{s}$

$5-70^{\circ} \mathrm{C}$ 
Table 4 Properties of the calibration fluids at $25^{\circ} \mathrm{C}$. The * indicates measurements carried out with an Ubbelohde

\begin{tabular}{lccl}
\hline Fluid & \multicolumn{2}{l}{$\begin{array}{l}\eta \\
{\left[\mathrm{kg} / \mathrm{m}^{3}\right]}\end{array}$} & $\begin{array}{l}\eta \\
{[\mathrm{mPas}]}\end{array}$ \\
\hline air & 1.17 & 0.0185 & Lide (1991) \\
hexane & 653 & 0.290 & Viswanath (1989) \\
toluene & 866 & 0.558 & Lide (1991) \\
carbon-tetrachlor & 1594 & 0.908 & Lide (1991) \\
ethanol & 785.9 & 1.074 & Lide (1991) \\
1-butanol & 807 & 2.57 & Lide (1991) \\
dibutylphthalate & 1041.9 & 16.2 & Lide (1991) \\
diethylhexylphthalate & 980.4 & 57.4 & $*$ \\
baysilon M 100 & 964.1 & 95.2 & $*$ \\
baysilon M350 & 967.4 & 329.9 & $*$ \\
baysilon M500 & 968.9 & 482.8 & $*$ \\
glycerole & 1258.8 & 906.0 & $*$ \\
baysilon M1000 & 969.5 & 938.5 & $*$ \\
\hline
\end{tabular}

with $C_{1}=M^{\prime} \omega_{s} / K, \quad C_{2}=U \omega_{s} / K$ and $C_{3}=D \omega_{s} / K$. Now the calibration procedure is as follows:

1) A set of Newtonian fluids is chosen as standards for the calibration. These fluids are listed in Table 4.

2) For each fluid, $R$ and $\varphi$ are measured as a function of the angular frequency. From a fit of $R \cos \varphi$ and $R \sin \varphi$ as a function of the frequency according to Eqs. (42a) and (42 b) the values for $\omega_{s}, C_{0}$ and $C_{1}$ are determined.

In Fig. $5 R \sin \varphi$ is plotted as a function of the frequency. Figure $5 \mathrm{a}$ shows in detail the approximate sinusoidal behavior from $0 \mathrm{up}$ to $50 \mathrm{~Hz}$ for a selection of the calibration fluids. As for different viscosities the value of $C_{0}$ is different, the slope at zero frequency of the curves increases with increasing viscosity. The lines represent the fits of Eq. (42b). For low viscosities the data points deviate from the fit at frequencies above approximately $10 \mathrm{~Hz}$ as can be seen in the enlarged Fig. 5 b. For these low viscous fluids the gap-loading limit does not hold anymore at these frequencies. No measurements have been carried out in the range $28-35 \mathrm{~Hz}$, because in this range scatter in the measurements occurs, probably due to a torsional resonance of the system.

In Fig. $6, R \cos \varphi$ is plotted as a function of the frequency for the same fluids. As the constants $\omega_{s}$ and $C_{1}$ do not depend on the viscosity this yields approximately a single curve to which all data can be fitted. The reason that not all data points lie on a single curve is due to the different densities which affect $M^{\prime}$. This is a small effect and can be taken into account, but will not be discussed here. The constant $\omega_{s}$ has been determined with Eqs. (42a) as well as (42b).

3) Repeating procedure 2 for all fluids gives information concerning the reproducibility of the constants $\omega_{s}$ and $C_{1}$. Furthermore, different values for $C_{0}$ are obtained for different fluids. As $C_{0}$ is of the form

$$
C_{0}=C_{2} \eta^{\prime}+C_{3}
$$

(a)

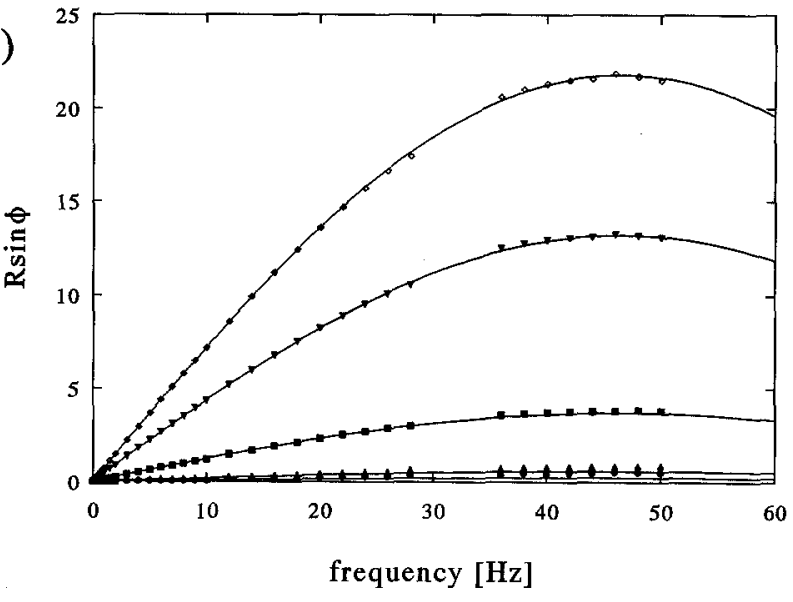

(b)

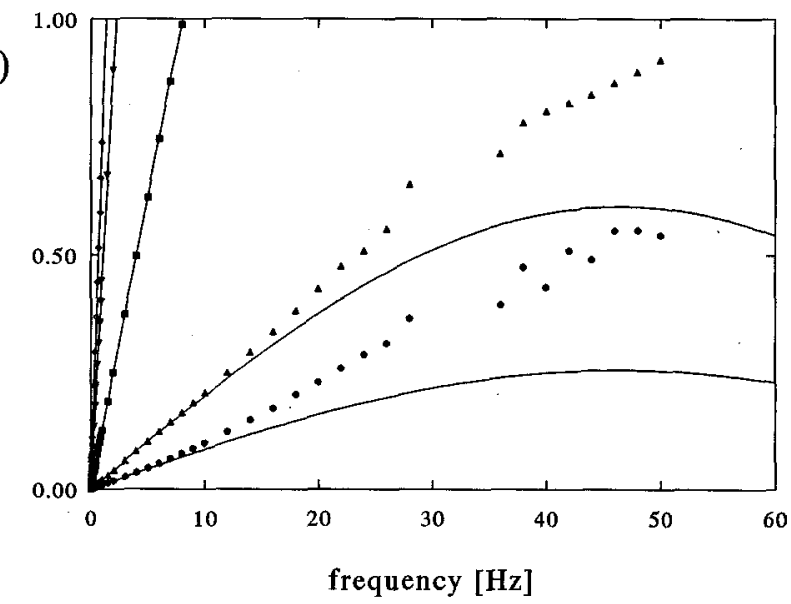

Fig. 5 a) $R \sin \varphi$ as a function of the frequency for the calibration fluids. b) $R \sin \varphi$ expanded $y$ axis. - Ethanol, $\Delta 1$-butanol, $\square$ dibutylphthalate, $\boldsymbol{\nabla}$ diethylhexylphthalate, $\bullet$ baysilon M100

and since the viscosity of the calibration fluids is known, a fit yields the constants $C_{2}$ and $C_{3}$ for the calibration fluids (see Fig. 7). The data point of air has not been used in the fit procedure because of its inaccuracy. The fit approximates all data points within $2 \%$ and holds for viscosities in the range from 0.1 to $1000 \mathrm{mPas}$.

4) Knowing the constants $C_{1}, C_{2}$ and $C_{3}$, the constants $K / U\left(=\omega_{s} / C_{2}\right), D / U\left(=C_{3} / C_{2}\right)$ and $M^{\prime} / U\left(=C_{1} / C_{2}\right)$, are calculated. The results are listed in Table 5 . Here it has to be remarked that it is not possible to calculate the physical constants $K, m, M^{\prime}, U$ and $D$ separately with this method, however, if one of these constants is determined from a different procedure, then it is possible to determine all the others. For instance the mass $M$ or the spring constant $K$ can be determined with a balance and $U$ can be calculated theoretically. If one of these values is inserted the others are found to be consistent with the dimensions of the instrument, and so with the calculated 
Fig. $6 R \cos \varphi$ as a function of the frequency the calibration fluids. - ethanol, A 1-butanol, $\square$ dibutylphthalate, $\boldsymbol{\nabla}$ diethylhexylphthalate, baysilon M100

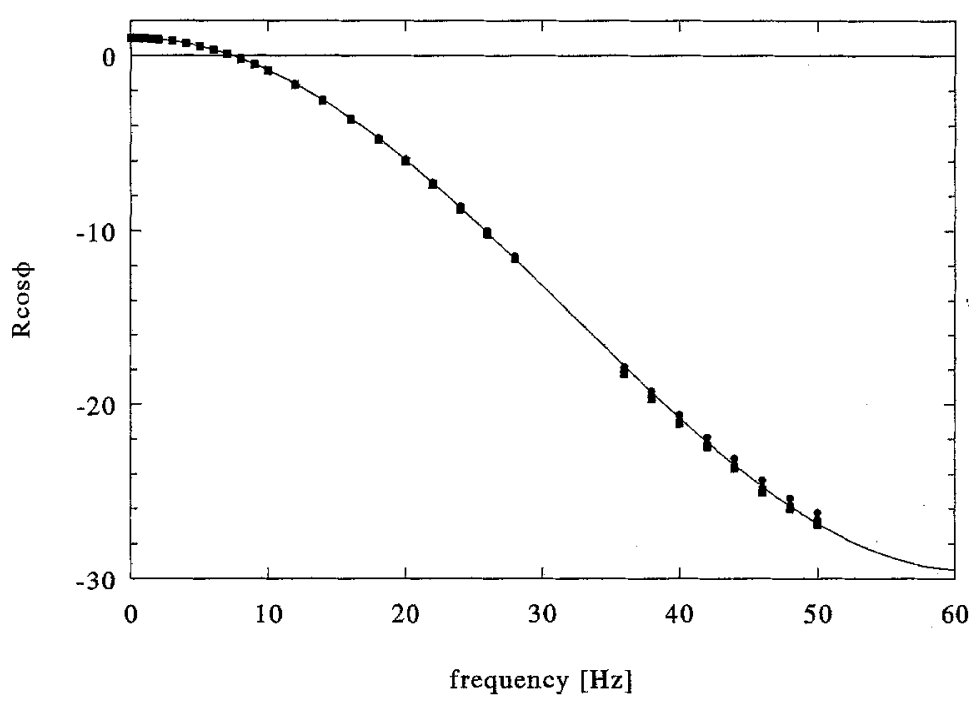

$$
\begin{aligned}
& \eta^{\prime}=\frac{K}{U} \frac{R \sin \varphi}{\omega_{s} \sin \left(\omega / \omega_{s}\right)}-\frac{D}{U} \\
& \eta^{\prime \prime}=\frac{K}{U} \frac{R \cos \varphi-\cos \left(\omega / \omega_{s}\right)}{\omega_{s} \sin \left(\omega / \omega_{s}\right)}+\frac{M^{\prime}}{U} \omega
\end{aligned}
$$

\section{Measurement range}

The measurement range depends both on the limits of applicability of Eq. (44) and the sensitivity of the LVDT's used. An impression of the validity of Eq. (44) can be given by considering the series expansion for the impedance $Z^{*}$. It is possible to calculate the change in viscosity ( $\left.\Delta \eta^{*}\right)$ if the $\alpha_{2}$ term is taken into account. From Eq. (28) it can be derived that
Fig. 7 Coefficient $C_{0}$ as a function of $\eta^{\prime}$. The $\square$ indicate the value obtained for air

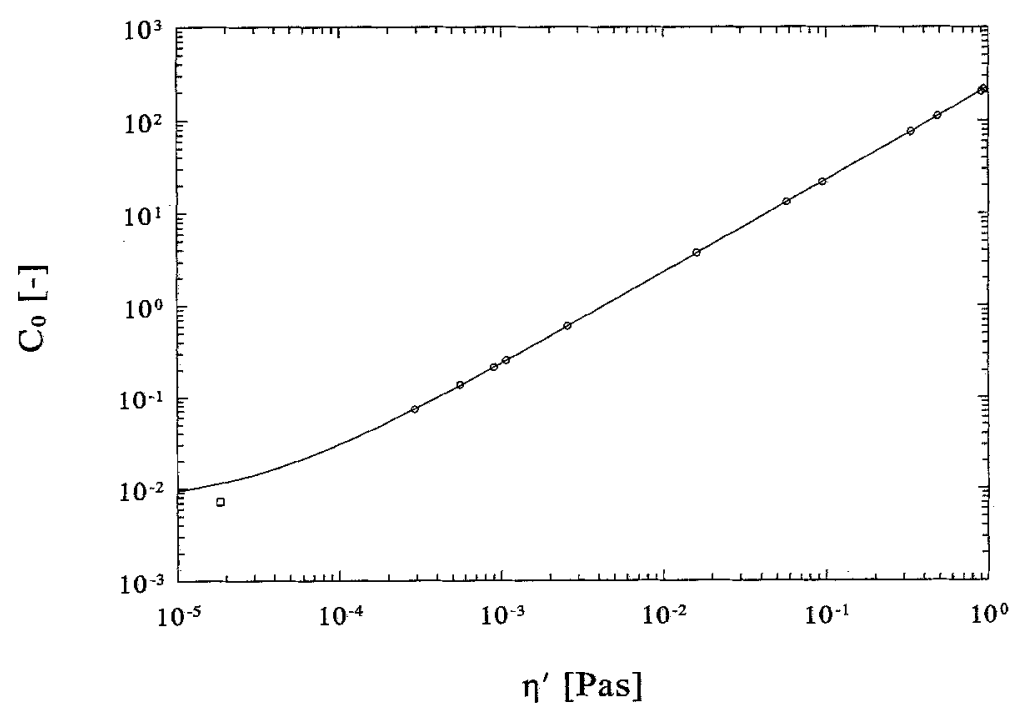




$$
Z^{*}=U\left(\eta^{*}+i a_{1}+a_{2} / \eta^{*}\right)
$$

with $a_{1}=\left(\alpha_{1} / \alpha_{0}\right) \rho \omega(b-a)^{2}$ and $a_{2}=\left(\alpha_{2} / \alpha_{0}\right) \rho^{2} \omega^{2}$ $(b-a)^{4}$.

The last term is the first higher order approximation term to be taken into account. So up to and including first order, it follows from Eq. (45) that

$$
\eta^{*}=\frac{Z^{*}}{U}-i a_{1}
$$

which is equivalent with Eq. (44). Taking into account the $a_{2}$ term it easily follows that the correction term becomes $\Delta \eta^{*}=-a_{2} / \eta^{*}$, so the relative error due to the gaploading approximation can be estimated:

$$
\left|\frac{\Delta \eta^{*}}{\eta^{*}}\right|=\frac{\left|a_{2}\right|}{\left|\eta^{*}\right|^{2}}
$$

If an accuracy of $|\Delta \eta * / \eta *| \leq \delta$ is required, the following equation holds:

$$
\left|\eta^{*}\right| \geq A v
$$

where $A=\left(\alpha_{2} / \alpha_{0}\right)^{1 / 2} 2 \pi \rho(b-a)^{2} / \delta$ and $v$ is the frequency. If a density of $\rho=10^{3} \mathrm{~kg} / \mathrm{m}^{3}$ is assumed and $\delta=10^{-2}$, then $A \simeq 5 \cdot 10^{-4} \mathrm{Pas} / \mathrm{Hz}$.

Apart from the systematic errors due to deviations from the gap-loading limit also the finite resolution of the detectors influences the accuracy of the values obtained for the viscosity. Studying the cross-correlation technique applied, one concludes that $R$ and $\varphi$ are to be considered as independent variables which can be determined from the experimental data up to an accuracy of

$$
\delta \varphi=\delta R / R=\left\{\frac{2}{n}\left(\left(\frac{\Delta \zeta_{m}}{\zeta_{m}^{(0)}}\right)^{2}+\left(\frac{\Delta \zeta_{c}}{\zeta_{c}^{(0)}}\right)^{2}\right)\right\}^{1 / 2},
$$

where $n$ is the number of points taken in the cross-correlation and $\Delta \zeta_{m}$ and $\Delta \zeta_{c}$ are the uncertainties in the measured values of the momentary exciter and cylinder displacement, respectively. $\Delta \zeta_{m}$ is assumed to have the same value for all sample points as is $\Delta \zeta_{c}$. From Eq. (44), one obtains for the uncertainty in $\eta^{\prime}$ and $\eta^{\prime \prime}$ :

$$
\begin{aligned}
\delta \eta^{\prime}= & K R /\left(U \omega_{s} \sin \left(\omega / \omega_{s}\right)\right) \\
& \cdot\left[\sin ^{2} \varphi(\delta R / R)^{2}+\cos ^{2} \varphi(\delta \varphi)^{2}\right]^{1 / 2} \\
\delta \eta^{\prime \prime}= & K R /\left(U \omega_{s} \sin \left(\omega / \omega_{s}\right)\right) \\
& \cdot\left[\cos ^{2} \varphi(\delta R / R)^{2}+\sin ^{2} \varphi(\delta \varphi)^{2}\right]^{1 / 2}
\end{aligned}
$$

and with Eq. (49):

$$
\begin{aligned}
& \delta \eta^{\prime}=K R /\left(U \omega_{s} \sin \left(\omega / \omega_{s}\right)\right) \delta R / R \\
& \delta \eta^{\prime \prime}=K R /\left(U \omega_{s} \sin \left(\omega / \omega_{s}\right)\right) \delta R / R,
\end{aligned}
$$

which leads, with $R=\zeta_{m}^{(0)} / \zeta_{c}^{(0)}$, to

$$
\begin{aligned}
\delta \eta^{\prime}= & \delta \eta^{\prime \prime}=\frac{K R}{U \omega_{s} \sin \left(\omega / \omega_{s}\right)} \frac{1}{\zeta_{m}^{(0)}} \\
& \cdot\left\{\frac{2}{n}\left(\left(\Delta \zeta_{m}\right)^{2}+R^{2}\left(\Delta \zeta_{c}\right)^{2}\right)\right\}^{1 / 2} .
\end{aligned}
$$

In principle, experiments are performed at constant $\gamma_{0}$ so $\zeta_{c}^{(0)}=c \cdot \gamma_{0}$ and $\zeta_{m}^{(0)}=R c \cdot \gamma_{0}$, where $c$ is a geometrical constant. But for $R>\zeta_{m m} /\left(c \cdot \gamma_{0}\right)$ in which $\zeta_{m m}$ is the maximal displacement of the exciter shaft, $\gamma$ will decrease: $\gamma=\zeta_{m m} /(R c)<\gamma_{0}$. With the equations given above, one is able to calculate $\delta \eta^{\prime}\left(\eta^{\prime}, \eta^{\prime \prime}, \omega, \gamma_{0}\right)$ once the values for $\Delta \zeta_{m}, \Delta \zeta_{c}$ and $n$ are known. For the instrument described, $\Delta \zeta_{m}=3 \cdot 10^{-7} \mathrm{~m}$ and $\Delta \zeta_{c} \simeq 3 \cdot 10^{-7} \mathrm{~m}$. These values have been obtained from the variations on the LVDT output signals as observed on a monitor scope. The number of data points sampled per period is about $n=1000$ for both signals. We calculated the relative error, $\delta \eta^{\prime} /\left|\eta^{*}\right|$, in the frequency range $0.001<\omega / 2 \pi<50 \mathrm{~Hz}$ for different values of $\gamma_{0}$ between 0.0032 and 3.2. In these ranges the calculated relative error depends on $\left|\eta^{*}\right|, \omega$ and $\gamma_{0}$ but not on $\eta^{\prime} / \eta^{\prime \prime}$. From this calculation the upper and lower bound of the viscosity as a function of the frequency have been obtained for which the accuracy of the results is better than $5 \%$ (see Fig. 8).

In Fig. 9 the calculated accuracy in $\eta^{\prime}$ and $\eta^{\prime \prime}$ according to Eq. (51), for diethylhexylphthalate is compared with the measured reproducibility for different values of $\gamma_{0}$. It can be observed that the calculated behavior is in agreement with the measurements.

\section{Results}

After the calibration of the instrument a number of tests and measurements was carried out. Here some frequency and shear rate-dependent results will be presented for Newtonian fluids as well as for a polystyrene solution in toluene and a dispersion of charged latex spheres in water.

Newtonian liquids

As a demonstration of the capabilities of the instrument measurements of a number of Newtonian liquids are shown. In Fig. 10 the complex viscosity of ethanol, 1-butanol, dibutylphthalate (DBP) and diethylhexylphthalate (DEHP) as a function of the frequency are given. For the low viscosities (Fig. 10a), the measurements start to 


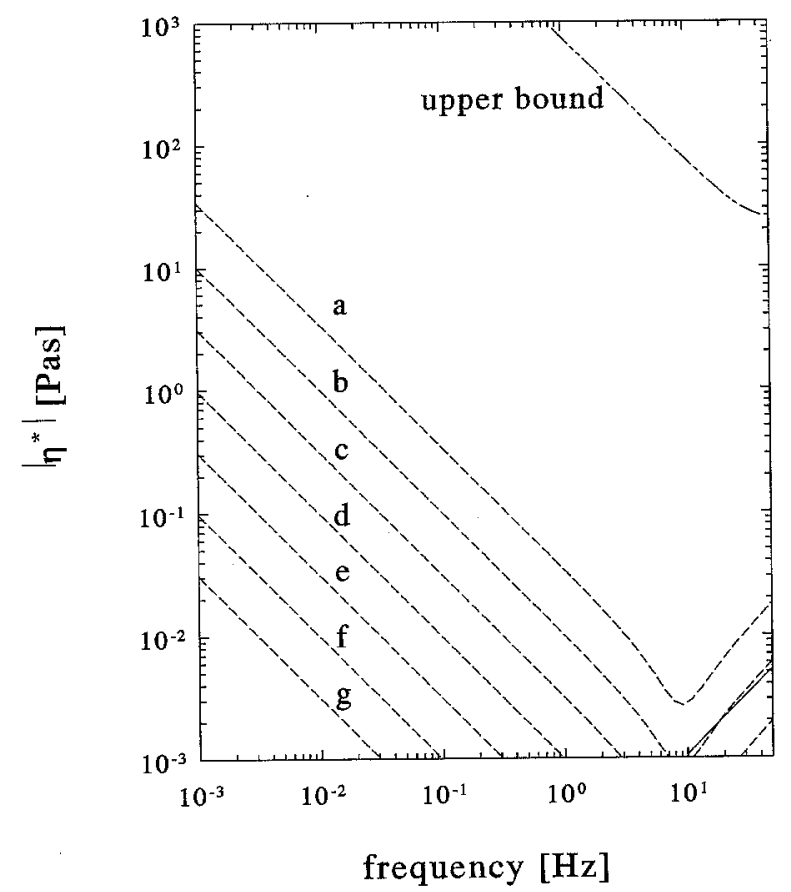

Fig. 8 The upper and lower bound for $\left|\eta^{*}\right|$ as a function of the frequency for which $\delta \eta^{\prime} /\left|\eta^{*}\right|<0.05 . \gamma_{0}=0.0032$ (a), 0.01 (b), $0.032(\mathrm{c}), 0.1(\mathrm{~d}), 0.32(\mathrm{e}), 1.0(\mathrm{f})$ and $3.2(\mathrm{~g})$. Also indicated is the gap-loading limit in the lower right corner (solid line)

deviate from the expected behavior at low frequencies $(v<0.02 \mathrm{~Hz})$ due to the finite resolution of the instrument and above about $15 \mathrm{~Hz}$ the gap-loading limit does not hold anymore. Moreover, a sharp resonance of the measuring system, probably due to the geometry of the spring used, occurs at $32 \mathrm{~Hz}$. For the higher viscosities this resonance is suppressed effectively and the applicable frequency range is much more extended (see Fig. 10b).
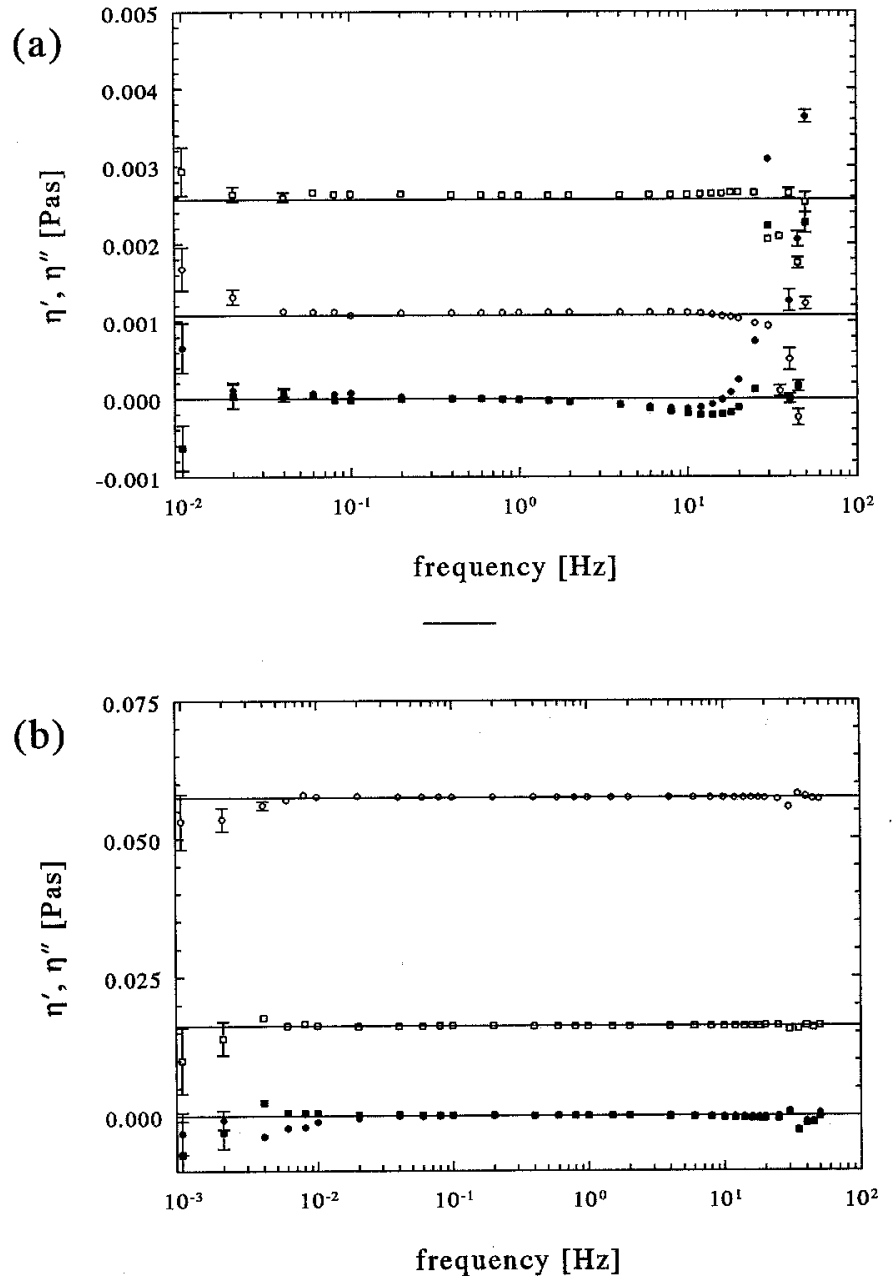

Fig. 10 The viscosity $\eta^{\prime}$ and $\eta^{\prime \prime}$ as a function of the frequency. a)

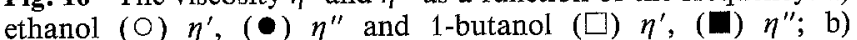
dibutylphthalate $(\square) \eta^{\prime}$, (回) $\eta^{\prime \prime}$ and diethylhexylphthalate $(0) \eta^{\prime}$, $(\bullet) \eta^{\prime \prime}$. Lines indicate the literature values
Fig. 9 Comparison between the measured and calculated uncertainty in the viscosity of DEHP for $\gamma_{0}=0.064(\nabla)$,

$0.32(\square), 0.64(\triangle)$ and 3.2 (O); open symbols: $\delta \eta^{\prime}$, solid symbols: $\delta \eta^{\prime \prime}$

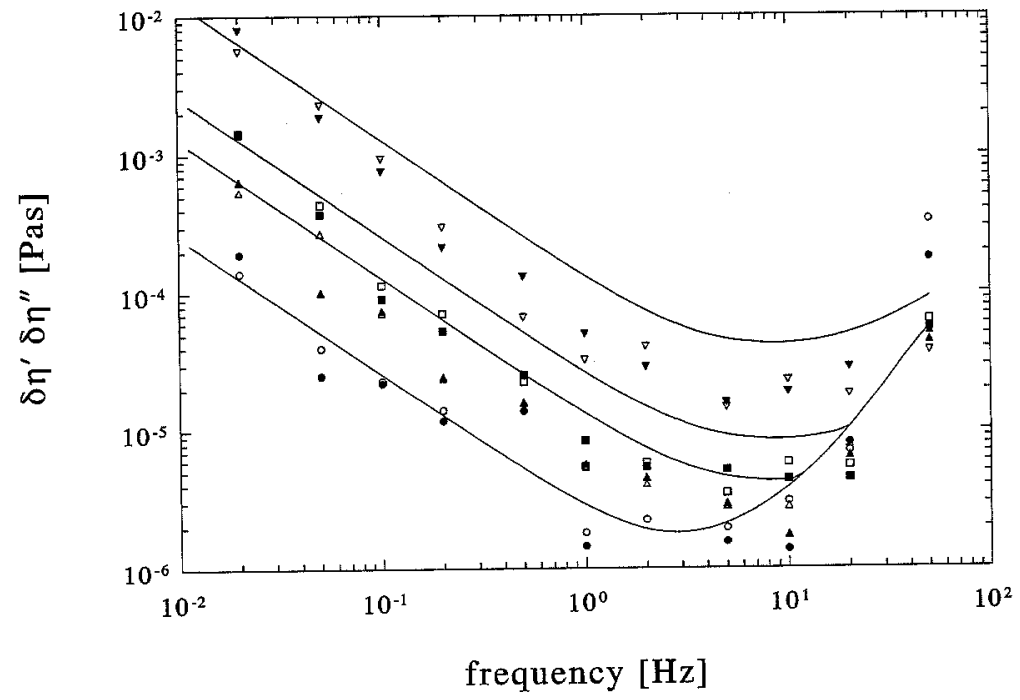


Fig. 11 Viscosity $\eta^{\prime}$ and $\eta^{\prime \prime}$ as a function of the frequency at $\dot{\gamma}=125 \mathrm{~s}^{-1}$ at $\gamma \cong 3.2,(0)$ $\eta^{\prime},(\bullet) \eta^{\prime \prime}$ and $\gamma \cong 0.064$, ( $\left.\square\right)$ $\eta^{\prime}(\mathbf{\square}) \eta^{\prime \prime}$. Lines indicate the literature values

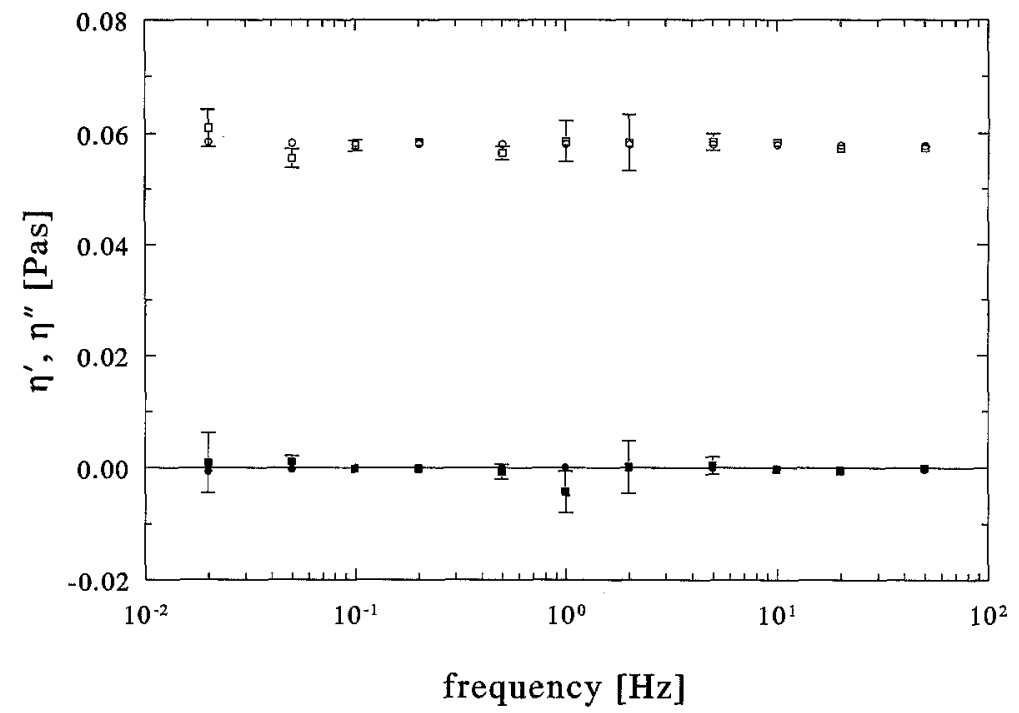

These measurements were performed with a deformation amplitude of $\gamma=3.2$. If necessary, measuring at low frequencies can be improved by using a spring with a lower spring constant.

In Fig. 11 measurements of the complex viscosity of DEHP as a function of the frequency are given, simultaneously shearing at a steady shear rate of $125 \mathrm{~s}^{-1}$. The measurements are done at two deformation amplitudes: $\gamma=3.2$ and $\gamma=0.064$, respectively. From this figure the linear behavior of the instrument can be observed.

The errors in the complex viscosity at the lower deformation amplitude are due to the resolution of the instrument except for the measurements at about $1 \mathrm{~Hz}$. These errors are caused by a small misalignment of the rotating drive shaft which supports the cup. At a shear rate of $125 \mathrm{~s}^{-1}$, the rotation frequency of the cup equals $1.5 \mathrm{~Hz}$ and in this frequency range the cross-correlation technique fails to filter out the rotatory disturbance.

\section{Polymer solution}

A solution of $0.6 \mathrm{wt} \%$ polystyrene in toluene is used as a demonstration of a $\eta_{\perp}^{*}(\dot{\gamma}, \omega)$ measurement of a viscoelastic and shear thinning liquid. The polystyrene with a molecular weight of $26 \cdot 10^{6}$ was obtained from Waters ass., Milford and the toluene, $99 \%$ pure, from Merck. In Fig. 12 the real and imaginary part of the complex viscosity, measured at a number of fixed frequencies has been plotted as a function of the applied shear rate. All measurements were performed in the linear viscoelastic regime. As can be seen in Fig. 12 both $\eta_{\perp}^{\prime}(\dot{\gamma}, \omega)$ and $\eta_{\perp}^{\prime \prime}(\dot{\gamma}, \omega)$ decrease with increasing shear rate.

The complex viscosity as a function of the frequency for a dilute polymer solution can be described by (Ferry, 1980)
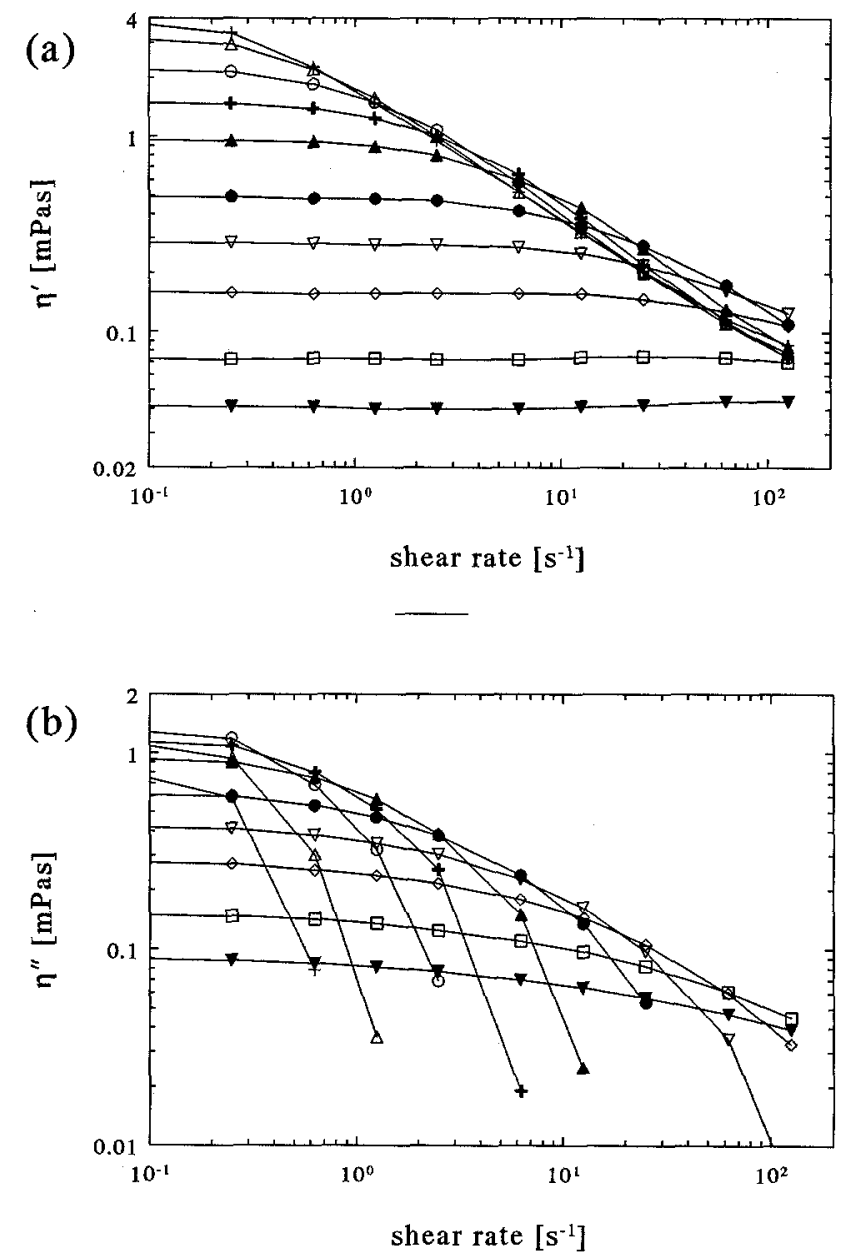

Fig. 12 Viscosity of polystyrene in toluene $1 \%$, a) $\eta^{\prime}$, b) $\eta^{\prime \prime}$ as a function of shear rate. Frequencies correspond with: $(+) 0.01 \mathrm{~Hz}$, $(\triangle) 0.02 \mathrm{~Hz},(\bigcirc) 0.05 \mathrm{~Hz},(+) 0.1 \mathrm{~Hz},(\triangle) 0.2 \mathrm{~Hz},(\bullet) 0.5 \mathrm{~Hz}$, $(\nabla) 1.0 \mathrm{~Hz},(\diamond) 2.0 \mathrm{~Hz},(\square) 5.0 \mathrm{~Hz}$ and $(\nabla) 10 \mathrm{~Hz}$ 
Fig. 13 The longest relaxation time of the polymer solution as a function of the shear rate

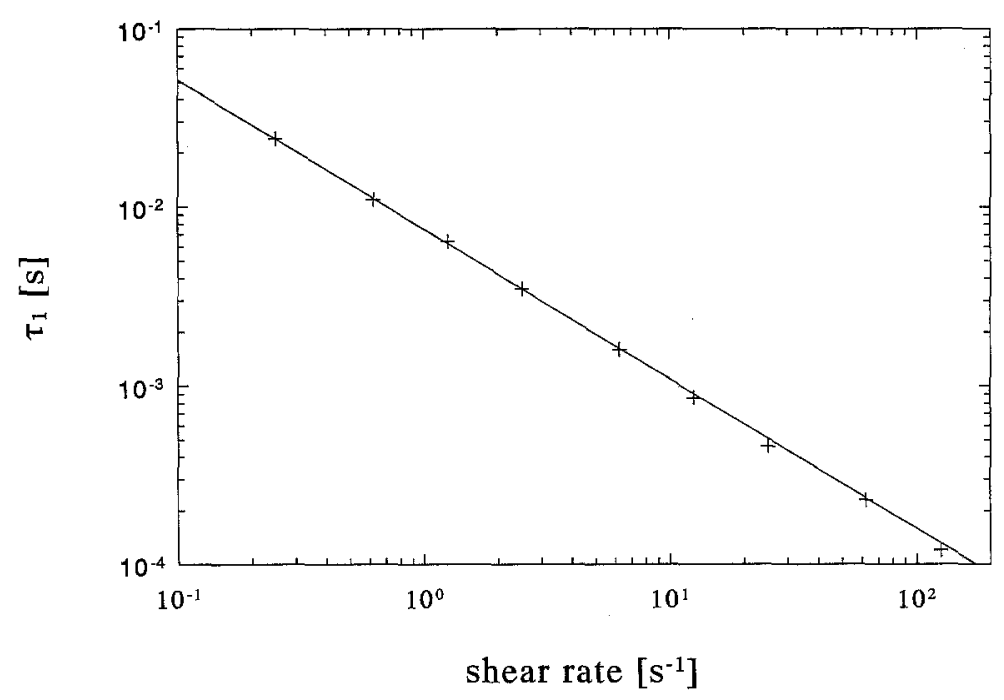

where $\eta_{\infty}^{\prime}$ is the real part of $\eta^{*}(\omega)$ at infinite frequency, $N$ the number of relaxation times involved and $G_{p}$ and $\tau_{p}$ are the relaxation strength and time, respectively. In case of Rouse-like behavior the relaxation times and strengths are coupled according to $\tau_{n}=\tau_{1} n^{-2}$ and $G_{n}=G_{1}$. The measurements of $\eta_{\perp}^{*}(\dot{\gamma}, \omega)$ at fixed shear rates can be fitted very well with this model. The resulting parameters will depend, of course, on the shear rate. The resulting longest relaxation time $\tau_{1}(j)$ as a function of the shear rate is given in Fig. 13. This dependence can be described as: $\tau_{1}(\dot{\gamma})=\tau_{1}(\dot{\gamma}=0) \cdot \exp (c \dot{\gamma})$ with $c=-0.84 \mathrm{~s}$. In the case of orthogonal superposition the limit $\eta_{\perp}^{*}(\dot{\gamma}, \omega \rightarrow 0)=\eta(\dot{\gamma})$ must hold. Indeed the $\eta^{\prime}(\dot{\gamma}, \omega=0.01 \mathrm{~Hz})$ curve in Fig. $12 \mathrm{a}$ resembles the $\eta(\dot{\gamma})$ behavior very closely: the same value for $\eta(j \rightarrow 0)$ and the same slope for $j>1 \mathrm{~s}^{-1}$.

\section{Ordered polystyrene latex}

Another example of $G_{\perp}^{*}(\dot{\gamma}, \omega)$ measurements on a complex fluid is given in Fig. 14. The investigated system is a monodisperse polystyrene latex dispersion in water. The volume fraction is 0.2 , the particle diameter is $490 \mathrm{~nm}$ and the surface charge density is about $-5 \mu \mathrm{C} / \mathrm{cm}^{2}$. The ionic strength of the water is about $10^{-5} \mathrm{~N}$. Due to the surface charge and the low ionic strength the particles order in a crystal lattice.

In Fig. 14 the real and imaginary part of the complex shear modulus as a function of the frequency at a number of fixed shear rates is given. As can be seen from this figure the behavior at zero shear rate is solid-like, e.g.,
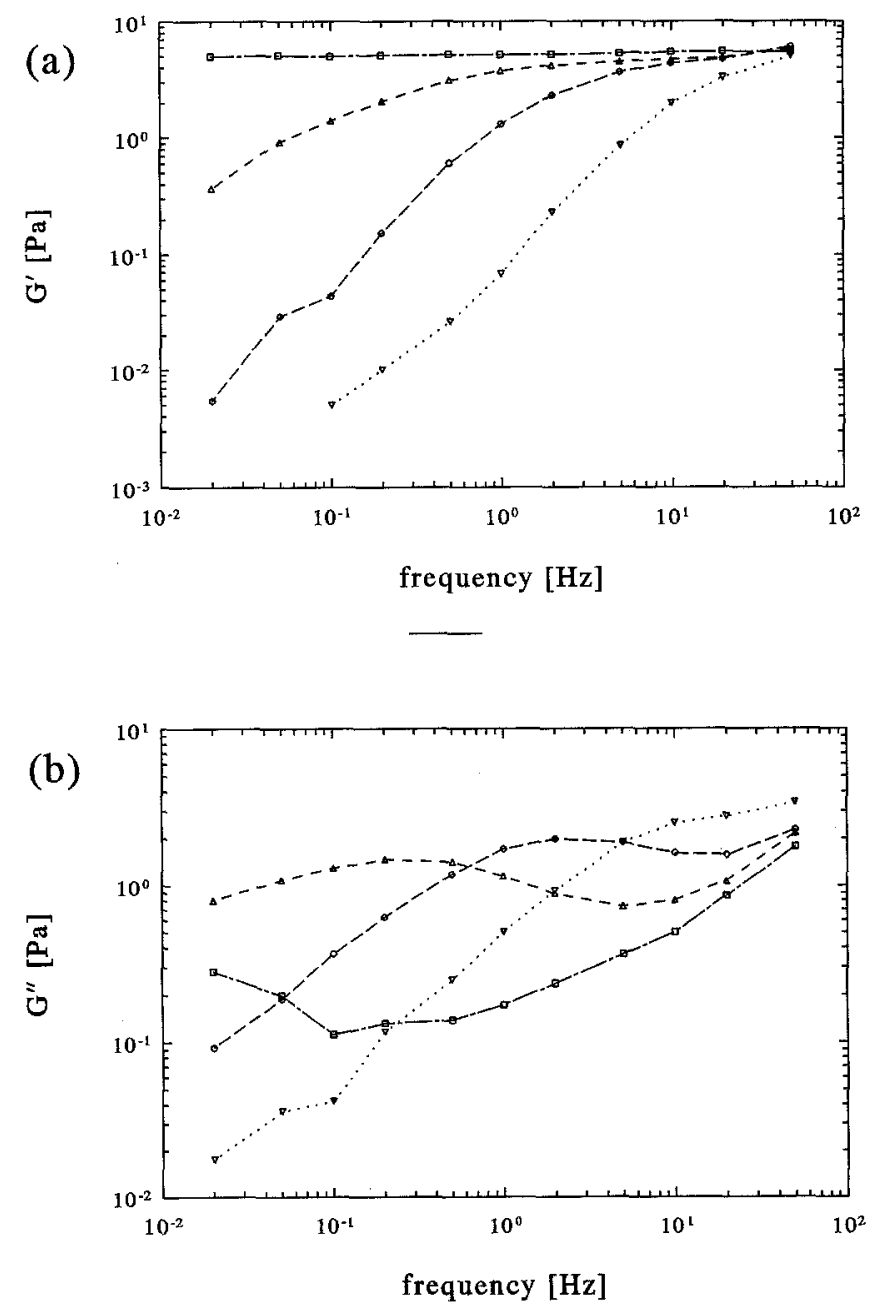

Fig. 14 The dynamic shear moduli of an ordering polystyrene latex dispersion at a volume fraction of $20 \%$ for $\dot{\gamma}=0.0(\square), 0.0625(\triangle)$, $0.625(0)$ and $6.25(\nabla) \mathrm{s}^{-1}$ 
$G^{\prime}(\dot{\gamma}=0, \omega)$ is almost independent of the frequency, while $G^{\prime \prime}(j=0, \omega)$ is much smaller than $G^{\prime}(j=0, \omega)$. With increasing shear rate the structure is gradually broken down, resulting in a lower and now frequencydependent storage modulus. Further analysis and discussion of these results will be published elsewhere.

\section{Conclusions}

A new instrument by which the viscoelastic properties of complex fluids can be measured has been developed. Measurements are carried out by applying a harmonic shear deformation. It is possible to apply simultaneously a small harmonic deformation orthogonal to a steady shear flow. The instrument has been calibrated for fluids in the range of $0.3 \mathrm{mPas}$ up to 1 Pas and in this range the calibration is accurate within $2 \%$. The sensitivity with respect to existing instruments has been improved as fluids with a typical viscosity of $1 \mathrm{mPas}$ can be measured at harmonic shear deformation frequencies down to $0.01 \mathrm{~Hz}$ and fluids with a viscosity of $20 \mathrm{mPas}$ and larger can be measured even down to $0.001 \mathrm{~Hz}$. Shear deformations as small as 0.005 can be applied and the steady shear rate can have a value from zero up to $125 \mathrm{~s}^{-1}$. The performance of the instrument is demonstrated with measurements on a number of Newtonian liquids as well as a viscoelastic polymer solution and an ordered latex dispersion. The measurements on the latter two systems show that interesting new phenomena concerning the flow behavior of complex fluids can be studied with this instrument.

\section{References}

Birnboim MH, Ferry JD (1961) Method for measuring dynamic mechanical properties of viscoelastic liquids and gels; the gelation of polyvinyl chloride. J Appl Phys 32:2305

Ferry JD (1980) Viscoelastic properties of polymers. John Wiley \& Sons, New York

Huilgol RR (1975) Continuum mechanics of viscoelastic liquids. Halsted Press, New York

Lide DR (1991) CRC Handbook of chemistry and physics $72^{\text {nd }}$ ed. Section 3 Physical constants of organic compounds. Section 6 Fluid properties, viscosity of liquids. CRC Press, Boca Raton

Markovitz H, A property of Bessel functions and its application to the theory of two rheometers. J Appl Phys 23:1070

Marin G (1988) Oscillatory rheometry. In: Collyer AA, Clegg DW (eds) Rheological measurement. Elsevier Applied Science, London
Mewis J, Schoukens G (1978) Mechanical spectroscopy of colloidal dispersions. Far Disc Chem Soc 535:58

Oka S (1960) The principles of rheometry. Rheology, theory and applications 3:17

Philippoff W (1934) Dynamische Untersuchungen an kolloiden Systemen. I Grundlagen und Methode. Phys Z 35:884

Pipkin AC, Owen DR (1967) Nearly viscometric flows. Phys of Fluids 10:836

Powell JW (1970) Design of aerostatic bearings. The Machinery Publishing Co. Ltd., London

Schowalter WR (1978) Mechanics of nonNewtonian fluids. Pergamon Press, Oxford

Simmons JM (1966) A servo controlled rheometer for measurement of the dynamic modulus of viscoelastic fluids. $\mathrm{J}$ Sci Instr 43:887
Smith TL, Ferry JD (1949) Measurements of the mechanical properties of polymer solutions by electromagnetic transducers. J Appl Phys 20:144

Tanner RI, Williams G (1971) On the orthogonal superposition of simple shearing and small strain oscillatory motions. Rheol Acta 10:528

Van den Ende D, Mellema J, Blom C (1992) Driven torsion pendulum for measuring the complex shear modulus in a steady shear flow. Rheol Acta 31:194

Viswanath DS, Natarajan G (1989) Data book on the viscosity of liquids. Hemisphere Publishing Corporation, New York

Whorlow RW (1992) Rheological Techniques, 2nd ed. Ellis Horwood 